\title{
Exponential Convergence for One Dimensional Contact Processes*)
}

\author{
Chen Jinwen ${ }^{* * *}$ (陈金文) \\ Department of Mathematics, Beijün Normal University \\ Richard Durrett **) \\ Cornell University, USA \\ Liu Xiufang ${ }^{* * *}$ (刘秀芳) \\ Department of Mathemutics. Beijing Normal University
}

Received March 23, 1988 Revised February 30, 1989

\begin{abstract}
The complete convergence theorem implies that starting from any initial distribution the one dimensional contact process converges to a limit as $t \rightarrow \infty$. In this paper we give a necessary and sufficient condition on the initial distribution for the convergence to occur with exponential rapidity.
\end{abstract}

\section{Introduction}

Since Harris (1974) introduced the contact process, many papers have been written on this topic, especially for the basic contact process in one dimension ( see [1], [4], [6], [7], and [8]). In this paper we will consider the one dimensional nearest neighbor case, that is, a Markov process $\xi, \subset \mathbb{Z}$ with:

$$
\begin{array}{ll}
P\left(x \in \xi_{t+s} \mid \xi_{1}\right)=\beta_{x}\left(\xi_{t}\right) s+o(s) & \text { when } x \notin \xi_{t}, \\
P\left(x \notin \xi_{t+s} \mid \xi_{t}\right)=\delta_{x}\left(\xi_{t}\right) s+o(s) & \text { when } x \in \xi_{t},
\end{array}
$$

where the birth and death rates are given by

$$
\beta_{x}(\xi)=\left\{\begin{array}{l}
0 \text { if }|\xi \cap\{x-1, x+1\}|=0, \\
\lambda \text { if }|\xi \cap\{x-1, x+1\}|=1, \\
\theta \lambda \text { if }|\xi \cap\{x-1, x+1\}|=2,
\end{array}\right.
$$

and $\delta_{x}(\xi)=1$. Here $\theta \geqslant 1$ is considered to be fixed while $\lambda>0$ is varied. When $\theta=2$ we get the basic contact process.

If $A \subset \mathbb{Z}$, let $\xi_{1}^{A}$ denote the process with $\xi_{0}^{A}=A$. We assume that $\theta \geqslant 1$ so the system is attractive: if $A \subset B$ then we can construct $\xi_{t}^{A}$ and $\xi_{t}^{B}$ on the same space with $\xi_{t}^{A} \subset \xi_{t}^{B}$ for all $t$. A consequence of attractiveness is that $\xi_{t}^{Z} \Rightarrow v$ as $t \rightarrow \infty$, where $\Rightarrow$ denotes the weak convergence, which in this setting is just the convergence of finite dimensional distributions. $v$ is a stationary distribution for the contact process, but may be the trivial one: $\delta \mid=a$ pointmass on $\phi$. Let $\lambda_{c}(\theta)=\inf \{\lambda: v \neq \delta \phi\}$. It is known that if $\theta \geqslant 1$ then $\lambda_{c}(\theta) \leqslant \lambda_{c}(1) \leqslant 4$, and that the following complete convergence theorem holds for $\lambda>\lambda_{c}(\theta)$ :

*) This work was discussed while the authors were visiting the Nankai Mathematics Institute in Tianjin.

**) Partially supported by the National Science Foundation, the Army Research Office through the Mathematical Sciences Institute at Cornell University, and a Guggenheim fellowship.

***) Research supported by the National Science Foundation of China. 
(*)

$$
\xi_{t}^{\mu} \Rightarrow P\left(\tau^{\mu}<\infty\right) \delta \phi+P\left(\tau^{\mu}=\infty\right) v .
$$

Here $\xi_{1}^{\mu}$ denotes the contact process with initial distribution $\mu$ and $\tau^{\mu}=\inf \left\{t: \xi_{t}^{\mu}=\phi\right\}$. This result was proved in [1] for $1 \leqslant \theta \leqslant 2$. Using Theorem 4 in [5] it is easy to extend the proof to $\theta>2$.

The purpose of this paper is to identify the initial distributions for which the convergence in $\left({ }^{*}\right)$ occurs with exponential rapidity, that is, for which the following conclusion holds:

(**) For any nonempty finite $B \subset \mathbb{Z}$, there are constants $C, y \in(0, \infty)$ such that

$$
\left|P\left(B \subset \xi_{t}^{\mu}\right)-P\left(\tau^{\mu}=\infty\right) v(\xi: \xi \supset B)\right| \leqslant C e^{-\gamma t} .
$$

Since any event involving finitely many coordinates can be written in terms of the events $\{\xi: B \subset \xi\},\left({ }^{* *}\right)$ gives the exponential convergence of finite dimensional dis tributions.

In [5] (see Theorem 2 on p. 383) it was shown that (**) holds when $\mu=\delta_{\varkappa \psi}$. In [6] ( see p. 172) it was shown that if $\mu$ is a product measure with density $p$, i.e.

$$
\mu(\xi: B \subset \xi)=p^{|B|},
$$

where $|B|=$ the number of points, then $(* *)$ holds when $\theta=2$ and $\lambda \geqslant 14$.

Before this work, the first and third authors showed that $(* *)$ holds for one dimensional supercritical contact processes (i. e. $\theta \geqslant 1, \lambda>\lambda_{c}(\theta)$ ) when $\mu=\delta_{A}$, where $A$ is a finite set or $A \supset k_{0} \mathbb{Z}_{+}$for some integer $k_{0}$ with $\left|k_{0}\right| \geqslant 1$. The next re sult shows that exponential convergence always holds for product measures and includes the case mentioned above.

Theorem. Let $\lambda>\lambda_{c}(\theta) .(* *)$ holds if and ony if there are constants $C, \delta$, $\gamma \in(0, \infty)$ such that for all $n \in \mathbb{Z}_{+}$,

$$
\mu\left(\xi:|\xi \cap[-n, n]| \leqslant \delta n, \xi \cap[-n, n]^{c} \neq \phi\right) \leqslant C e^{-\gamma n} .
$$

Before explaining the intuition behind the theorem, we need to state some known results:

(1.1) Let $\tau^{A}=\inf \left\{t: \xi_{t}^{A}=\phi\right\}$. There are $C, \gamma \in(0, \infty)$ such that for all $t \geqslant 0$ and $A \subset \mathbb{Z}, P\left(t<\tau^{A}<\infty\right) \leqslant C e^{-\gamma t}$.

(1.2) There are $C, \gamma \in(0, \infty)$ such that $P\left(\tau^{A}<\infty\right) \leqslant C e^{-\gamma|A|}$ for all $A \subset \mathbb{Z}$.

(1.3) Let $r_{t}^{A}=\sup \xi_{t}^{A}, r_{t}^{-}=r_{t}^{(-\infty, 0 l}$, and $\alpha(\lambda)=\lim E r_{t}^{-} / t$, which is $>0$ for $\lambda>\lambda_{c}(\theta)$. Then for any $a<\alpha$ and $b>\alpha$ there are $C, \gamma \in(0, \infty)$ such that for all $t \geqslant 0$,

$$
P\left(r_{t}^{-} \leqslant a t\right) \leqslant C e^{-\gamma t} \text { and } P\left(r_{t}^{-} \geqslant b t\right) \leqslant C e^{-\gamma t} .
$$

These conclusions were proved first for $1 \leqslant \theta \leqslant 2$ in [4] and extended to $\theta>2$ in [5]. Here and in what follows $C, \gamma$ denote positive finite constants whose values are unimportant and will change from line to line.

To explain why the condition is necessary we begin by considering what happens when $\mu(\xi:|\xi|=\infty)=1$ and hence $\xi_{t}^{\mu} \Rightarrow v$. In this case if $\mu(\xi: \mid \xi \cap[-n$, $n] \mid \leqslant \delta n) \geqslant e^{-\varepsilon n}$ then with probability at least $e^{-\varepsilon n} e^{-\Gamma \delta n}$ all the particles in $[-n, n]$ die by time 1 without giving birth and it follows from (1.3) that with probability at least $e^{-\varepsilon n} e^{-\Gamma \delta n}-2 C e^{-\gamma n / 2 \alpha}$ there will be no particles in $[-n / 3, n / 3]$ at time 
$t=n / 2 \alpha$.

The second part of the condition $\xi \cap[-n, n]^{c} \neq \phi$ is needed to take care of finite initial configurations. Our theorem implies that for a fixed finite initial configuration exponential convergence always occurs. If for example $\xi_{0}$ is a single particle at $X_{0}$, reasoning as in the last paragraph shows that exponential convergence occurs if and only if $P\left(\left|X_{0}\right|>n\right) \leqslant C e^{-\gamma n}$.

The proof of sufficiency, given in Section 2, is more technical. The key to the proof is a coupling result given in (2.1), from which the conclusion follows in a straightforward manner by using (1.1)-(1.3). The proof of necessity is given in Section 3.

\section{Proof of Sufficiency}

We begin by constructing the process. Define independent Poisson processes $\left\{S_{n}^{x}: n \geqslant 1\right\},\left\{T_{n}^{x}: n \geqslant 1\right\}$, and $\left\{U_{n}^{x}: n \geqslant 1\right\}$ for each $x \in \mathbb{Z}$ with rates $1, \lambda$, and $(\theta-1) \lambda$ respectively. As the reader can probably guess from the rates, at times

$S_{n}^{x}$ we kill a particle at $x$ if one is present,

$T_{n}^{x}$ a particle is born at $x$ if $x-1$ or $x+1$ is occupied,

$U_{n}^{x}$ a particle is born at $x$ if $x-1$ and $x+1$ are both occupied.

It is easy to see that using this "graphical representation" we can construct for each $\mu$ and $s$ the process starting from distribution $\mu$ at time $s:\left\{\xi_{t}^{\mu \cdot s} ; t \geqslant s\right\}$. See [5] for more details. In what follows it will be useful to use also the coordinate notation for our processes: $\xi_{t}^{\mu, s}(x)=1$ if $x \in \xi_{t}^{\mu \cdot s},=0$ otherwise.

To prove that our condition is sufficient we will prove several lemmas. The first one is a coupling property that is a special property of the nearest neighbor case. Let $l_{t}^{A}=\inf \xi_{t}^{A}$.

(2.1) Lemma. Let $E_{t}=\left\{r_{t}^{(-\infty,-a t]} \geqslant b t\right\}, F_{t}=\left\{l_{t}^{[a t, \infty)} \leqslant-b t\right\}, G_{t}=\left\{\tau^{A \cap[-a t, a t]}>t\right\}$, where $a, b>0$. On $E_{t} \cap F_{t} \cap G_{t}, \xi_{t}^{A}(x)=\xi_{t}(x)$ for $x \in[-b t, b t]$.

Proof. This follows easily from the proof of Lemma 13 in [5].

Pick $\varepsilon<\alpha / 4$, and let $a=(\alpha-2 \varepsilon)$ and $b=\varepsilon$. It follows from (1.3), translation invariance, and symmetry that

$$
P\left(l_{t}^{(a t, \infty)}>-\varepsilon t\right)=P\left(r_{t}^{(-\infty,-a t]}<\varepsilon t\right) \leqslant C e^{-\gamma t} .
$$

If $\mu$ satisfies the hypothesis of our theorem, then there are $\eta, C, \gamma \in(0, \infty)$ such that

$$
\mu\left(\xi:|\xi \cap[-a t, a t]| \leqslant \eta t, \xi \cap[-a t, a t]^{c} \neq \phi\right) \leqslant C e^{-\gamma t} .
$$

With (2.1)-(2.3) being established the rest is straightforward. Let

$$
\begin{gathered}
M_{t}=\{\xi:|\xi \cap[-a t, a t]|>\eta t\}, N_{t}=\{\xi \subset[-a t, a t]\}, \\
p(t)=\left|\int\left\{P\left(B \subset \xi_{t}^{A}\right)-P\left(\tau^{A}=\infty\right) v(\xi: B \subset \xi)\right\} \mu(d A)\right|,
\end{gathered}
$$

and for $i=1,2,3$ let

$$
p_{i}(t)=\int_{\Omega_{i}}\left|P\left(B \subset \xi_{i}^{A}\right)-P\left(\tau^{A}=\infty\right) v(\xi: B \subset \xi)\right| \mu(d A),
$$


where $\Omega_{1}=M_{t}^{c} \cap N_{t}^{c}, \Omega_{2}=M_{t}$, and $\Omega_{3}=N_{t}$. Clearly $p(t) \leqslant p_{1}(t)+p_{2}(t)+p_{3}(t)$. Since the integrand is a difference of two probabilities, it is $\leqslant 1$ and (2.3) implies

$$
p_{1}(t) \leqslant C e^{-\gamma t} .
$$

For the second term we observe

$$
p_{2}(t) \leqslant \int_{M_{t}}\left\{\left|P\left(B \subset \xi_{t}^{A}\right)-v(\xi: B \subset \xi)\right|+P\left(\tau^{A}<\infty\right)\right\} \mu(d A) .
$$

If $A \in M_{t}$ then $P\left(\tau^{A \cap \mid-a t, a d t}<\infty\right) \leqslant C e^{-\gamma t}$ by $(1.2)$; so it follows from (2.2) and (2.1) that $p_{2}(t) \leqslant C e^{-\gamma t}$. To bound the third term we observe

$$
\begin{aligned}
p_{3}(t) \leqslant & \int_{N_{t}}\left\{\left|P\left(B \subset \xi_{t}^{A}\right)-P\left(t / 2<\tau^{A}\right) P\left(B \subset \xi_{t / 2}^{L}\right)\right|+P\left(t / 2<\tau^{A}<\infty\right)\right. \\
& \left.+\left|P\left(B \subset \xi_{t / 2}^{4}\right)-v(\xi: B \subset \xi)\right| P\left(\tau^{A}=\infty\right)\right\} \mu(d A) .
\end{aligned}
$$

The last two terms in the integrand are $\leqslant C e^{-\gamma t}$ by (1.1) and the fact that exponential convergence holds for $\mu=\delta_{4}$. To estimate the first term, we observe that

$$
\begin{aligned}
& \left|P\left(B \subset \xi_{t}^{A}\right)-P\left(t / 2<\tau^{A}\right) P\left(B \subset \xi_{t / 2}^{Z}\right)\right| \\
= & \left|P\left(B \subset \xi_{t}^{A}, \tau^{A}>t / 2\right)-P\left(B \subset \xi_{t}^{Z, t / 2}, \tau^{A}>t / 2\right)\right|
\end{aligned}
$$

because $B \subset \xi_{t}^{2, t / 2}$ and $\tau^{\mathrm{A}}>t / 2$ are independent. When $A \in N_{t}, A \subset[-a t, a t]$ so (2.1) implies the last difference is smaller than

$$
P\left(t / 2<\tau^{A} \leqslant t\right)+P\left(E_{t}^{c}\right)+P\left(F_{t}^{c}\right) .
$$

The last quantity is $\leqslant C e^{-\gamma t}$ by $(1.1)$ and (2.2), and the proof is complete.

\section{Proof of Necessity}

The main step in proving necessity is to establish

(3.1) Lemma. Suppose $|A \cap[-n, n]| \leqslant \delta n$ and $A \cap[-n, n]^{c} \neq \phi$. Let $t=n / 2 \alpha$.

Then

$$
P\left(\tau^{A}>t / 2\right) P\left(0 \in \xi_{t}^{z, r / 2}\right)-P\left(0 \in \xi_{\tau}^{A}\right) \geqslant K e^{-(\theta \lambda+1) \delta n}-2 P\left(r_{t}^{-} \geqslant 2 \alpha t\right),
$$

where $K=e^{-1} P\left(\tau^{0}=\infty\right) v(\xi: 0 \in \xi)$.

Note. Of course, $P\left(r_{t}^{-} \geqslant 2 \alpha t\right) \leqslant C e^{-\gamma t}$ by $(1.3)$. We have written the result in the above form to emphasize that the error term, $-2 P\left(r_{t}^{-} \geqslant 2 \alpha t\right)$, does not depend on $\delta$. birth \},

Proof. Let $B_{n}=\{$ All particles in $A \cap[-n, n]$ die by time 1 and do not give

$$
\begin{gathered}
D_{n}=\left\{r_{t}^{(-\infty,-n]}<0, \quad l_{t}^{[n, \infty)}>0\right\} \\
x_{n}=\text { the point in } A \cap[-n, n]^{c} \text { closest to } 1 / 3 .
\end{gathered}
$$

Since $\tau^{A}>t / 2$ and $0 \in \xi^{2}, t^{\prime 2}$ are independent, we have

where

$$
P\left(\tau^{A}>t / 2\right) P\left(0 \in \xi_{t}^{\xi, t / 2}\right)-P\left(0 \in \xi_{t}^{A}\right)=p_{1}(t)+p_{2}(t)+p_{3}(t),
$$

$$
p_{1}(t)=P\left(\tau^{A}>t / 2,0 \in \xi_{t}^{Z \cdot t / 2}\right)-P\left(\tau^{A}>t / 2,0 \in \xi_{t}^{Z_{1} / 2}, B_{n}^{c}\right),
$$




$$
\begin{aligned}
& p_{2}(t)=P\left(\tau^{A}>t / 2,0 \in \xi_{t}^{4 / 2}, B_{n}^{c}\right)-P\left(\tau^{A}>t / 2,0 \in \xi_{t}^{2, t / 2}, B_{n}^{c} \cup D_{n}^{c}\right), \\
& p_{3}(t)=P\left(\tau^{A}>t / 2,0 \in \xi_{t}^{t, t / 2}, B_{n}^{c} \cup D_{n}^{c}\right)-P\left(\tau^{A}>t / 2,0 \in \xi_{t}^{A}\right) .
\end{aligned}
$$

On $B_{n} \cap D_{n}$ or $\left\{0 \notin \xi_{i}^{2, t / 2}\right\}, 0 \notin \xi_{t}^{A}$ so $p_{3}(t) \geqslant 0$. Clearly

$$
p_{2}(t) \geqslant-P\left(D_{n}^{c}\right) \geqslant-2 P\left(r_{t}^{-} \geqslant n\right) \text {, }
$$

by translation invariance. As for the remaining term,

$$
\begin{aligned}
p_{1}(t) & =P\left(\tau^{A}>t / 2,0 \in \xi_{t}^{\varkappa, t / 2}, B_{n}\right) \\
& \geqslant P\left(\text { the particle at } x_{n} \text { does not die by time } 1, \xi_{t / 2}^{x_{n}, 1} \neq \phi, 0 \in \xi_{t}^{\ll, t / 2}, B_{n}\right) \\
& \geqslant e^{-1} P\left(\tau^{0}=\infty\right) v(\xi: 0 \in \xi)\left\{e^{-\theta \lambda}\left(1-e^{-1}\right)\right\}^{|A \cap\{-n, n]|},
\end{aligned}
$$

since the four events are independent. Replacing $1-e^{-1}$ by $e^{-1}$ gives the desired bound.

to prove necessity now we write

$$
P\left(\tau^{\mu}=\infty\right) v(\xi: 0 \in \xi)-P\left(0 \in \xi_{t}^{\mu}\right)=q_{1}(t)+q_{2}(t)+q_{3}(t),
$$

where

$$
\begin{aligned}
& q_{1}(t)=P\left(\tau^{\mu}=\infty\right) v(\xi: 0 \in \xi)-P\left(\tau^{\mu}>t / 2\right) v(\xi: 0 \in \xi), \\
& q_{2}(t)=P\left(\tau^{\mu}>t / 2\right) v(\xi: 0 \in \xi)-P\left(\tau^{\mu}>t / 2\right) P\left(0 \in \xi_{t}^{\alpha, t / 2}\right), \\
& q_{3}(t)=P\left(\tau^{\mu}>t / 2\right) P\left(0 \in \xi_{t}^{\mathbb{L}, t / 2}\right)-P\left(0 \in \xi_{t}^{\mu}\right) .
\end{aligned}
$$

By (1.1), $q_{1}(t) \geqslant-C e^{-\gamma t}$. Exponential convergence for the case $\mu=\delta_{2}$ implies that

$$
q_{2}(t) \geqslant-C e^{-\gamma t}
$$

Notice that in both cases the constants $C, \gamma$ do not depend on $\mu$. Using (3.1) on the third term we see that

$$
q_{3}(t) \geqslant K e^{-(\theta \lambda+1) \delta n} \mu\left(A:|A \cap[-n, n]|<\delta n, A \cap[-n, n]^{c} \neq \phi\right)-2 P\left(r_{t}^{-} \geqslant 2 \alpha t\right)
$$

and the proof of necessity is complete.

\section{References}

[1] Durrett, R., On the growth of one dimensional contact processes, Ann. Prob., 8 (1980), $890-907$.

[ 2] Durrett, R., Oriented percolation in two dimensions, Ann. Prob., 12 (1984), 999 - 1040.

[ 3 ] Durrett, R., Lecture Notes on Particle Systems and Percolation, Wadsworth Pub. Co., Pacific Grove CA, 1988.

[ 4] Durrett, R. and Griffeath, D., Supercritical contact processes on $Z, A n n$. Prob., 11 (1983), $1-15$.

[ 5] Gray, L., Duality for general attractive spin systems with application in one dimension, Ann. Prob., 14 (1986), $371-396$.

[ 6] Griffeath, D., The basic contact process, Stoch. Processes Appl ., 11 (1981), $151-185$.

[7] Harris, T. E., Contact interactions on a lattice, Ann. Prob., 2 (1974), 969- 988.

[ 8 ] Liggett, T. M., Interacting Particle Systems, Springer Verlag, New York, 1985. 